\title{
Texture-Based Multiscale Segmentation: Application to Stromal Compartment Characterization on Ovarian Carcinoma Virtual Slides
}

\author{
Nicolas Signolle ${ }^{1,2}$, Benoît Plancoulaine ${ }^{2}$, Paulette Herlin², \\ and Marinette Revenu ${ }^{1}$ \\ ${ }^{1}$ GREYC ENSICAEN UMR 6072 6, Bvd Maréchal Juin 14050 CAEN France \\ 2 GRECAN EA 1772, iFR 146 ICORE, Université de Caen, CLCC François Baclesse \\ 2, Avenue du Général Harris 14076 CAEN cedex 5 France \\ nicolas.signolle@greyc.ensicaen.fr
}

\begin{abstract}
A multiscale segmentation strategy using wavelet-domain hidden Markov tree model and pairwise classifiers selection is tested in the present paper for histopathology virtual slide analysis. The classifiers selection is based on a study of the influence of hyper-parameters of the method. Combination of outputs of selected classifiers is then done with majority vote. The results of the segmentation of various types of stroma of ovarian carcinomas are presented and discussed.
\end{abstract}

\section{Introduction}

It is often necessary to identify and quantify cell and tissue compartments on histological sections, to estimate the potential evolution of the cancer lesions. To perform this measure, in an objective way, an image of the whole preparation (so-called Virtual Slide, VS) must be recorded and fully analyzed, as structures of interest are often heterogeneously spread all over the slide. It is also mandatory to adapt the working resolution to the size of the structures to be measured. High resolution VS produced for the identification of tiny microscopical structures occupy a very large volume in memory (several GigaBytes) and cannot be processed at once. Up to now, works performed on these large size images are scarce [12]. The method chosen by our team is a multiscale analysis of a high resolution $V S$, allowing to adapt the working resolution to the various structures to be segmented (cancer lesion, intra tumoral tissue compartments, cells and intra cellular structures for example).

The present study aims first at partitioning cancer cells and intra tumoral connective tissue and then at differentiating the various stromal compartments (fibrous tissue, loose mesenchymatous connective tissue, inflammatory cell accumulation). Stromal compartments can be identified mainly by their cell shape and organization. Then, each stromal component must exhibit an individual pattern which has to be characterized by texture analysis.

A. Elmoataz et al. (Eds.): ICISP 2008, LNCS 5099, pp. 173 182, 2008.

(C) Springer-Verlag Berlin Heidelberg 2008 
Many studies have been conducted on the segmentation of textured images. One can distinguish three categories of methods: the statistical methods which exploit the spatial distribution of the grey levels, the methods based on the construction of a texture model, and the methods which mimic the mechanism of the human vision, integrating several levels of resolution [3].

We chose to use the Hidden Markov Tree (HMT) proposed by Crouse [4 that combines these different approaches. It allows a statistical modeling of intra-scale and interscale properties of coefficients obtained by wavelet transform (WT) [5], and it exploits the decorrelation power of this transform. The goal is to capture the interscale dependency factor and the non-gaussian distribution of the coefficients computed at each scale. This model is applied to the segmentation of the various compartments of stroma identified inside VS of ovarian carcinomas, recorded at a high resolution $(0.5 \mu \mathrm{m})$.

The paper is organized as follows : because of the complexity and variability of the structures to be segmented, the "domain objects" are first precisely described, the mathematical background of the used tools is then detailed, the results are presented and discussed before concluding on the offered prospects.

\section{Material}

When a patient undergoes surgery for tumour resection, the excised biopsy is fixed in formalin, paraffin embedded and splitted off in $5 \mu \mathrm{m}$ thick slices. Slices are then affixed on a glass slide prior to be stained by imunohistochemistry or histochemistry. The special staining aims at revealing particular cell types or proteins which amount can then be estimated in order to assess the potential evolution of the tumour and to help choosing the best therapy protocol. In the case of ovarian carcinoma [Fig[1] the prognostic impact of the proportion of stroma is demonstrated [6]. The researchers want to further clarify the participation of the various stromal compartments in the evolution of tumours and their response to therapy.

The ovarian cancer lesion is made of two types of tissue: cancer epithelial cells and stroma. Stroma refers to the connective tissue which provides supportive framework and nutriments to epithelial cancer cells.

After hematoxylin staining, several types of stromal tissue can be identified inside tumours, some of which corresponding to various maturation degrees: loose connective tissue, cellular stroma and fibrous connective tissue. Inflammatory cell riched foci can also be found [Fig 2 .

- The loose connective tissue is an immature stroma. It is made of an abundant extracellular matrix and star shaped mesenchymatous cells. The density of these cells is low. Cell nuclei are small and round.

- The cellular stroma is a young connective tissue. It consists mainly of myofibroblasts organized in sheets and bundles with a high density of cells. The cell nuclei are elongated and plump. 

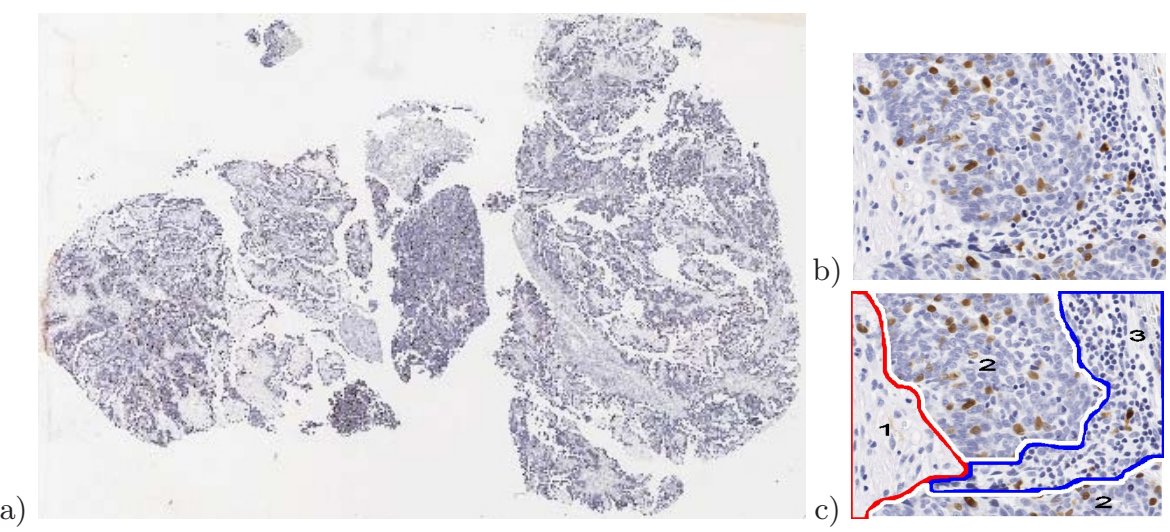

Fig. 1. a) Image of an histological section of ovarian carcinoma after DAB immunostaining of proliferation cells (brown) and Hematoxylin counterstaining of non proliferating nuclei (blue). The original size of the recorded image is 62000x41404 pixels (acquisition resolution $0.5 \mu \mathrm{m}$ ). b) Small detail. c) Manual drawing of the ideal segmentation of three compartments which can be identified on this detail ( 1 is loose connective tissue, 2 is cancer cell foci and 3 is inflammatory compartment).

- The fibrous connective tissue is a mature stroma with a density of cells somewhat lower than the cellular stroma and a large number of extra-cellular collagen fibers. The nuclei are very thin and elongated. Their optical density is high, due to chromatin condensation.

- The inflammatory stromal compartment is characterized by small round nuclei with a high optical density (lymphocytes and plasma cells) and clover shaped dense nuclei (polymorphonuclear cells).

Epithelium appears as blue (non proliferating) and brown (proliferating) cells with large size nucleus. Extravased red blood cells, necrosis foci and mucus sheets can also be found inside the tumor [Fig 2 .

Tiled tiff $V S$ were recorded at a resolution of $0,5 \mu \mathrm{m}$ thanks to a high resolution slide scanner (Scanscope CS from Aperio Technologies) provided with a 20x objective. A 30\% loss Jpeg compression was applied to each tile when recording images. Uncompressed Images are 3 to 10 GigaBytes files, depending on the scanned tissue area.

\section{Methods}

\subsection{Multiscale Strategy}

Previous works based on low resolution analysis strategy $(6.3 \mu \mathrm{m})$ already allow to segment and quantify markers (quantification of stained nucleus, of epithelium, of blood vessels, of stroma [7/8]). However, the fine analysis of stroma compartments, which needs a cell characterization, is impossible at this scale as a cell nucleus is represented by only a few pixels. 


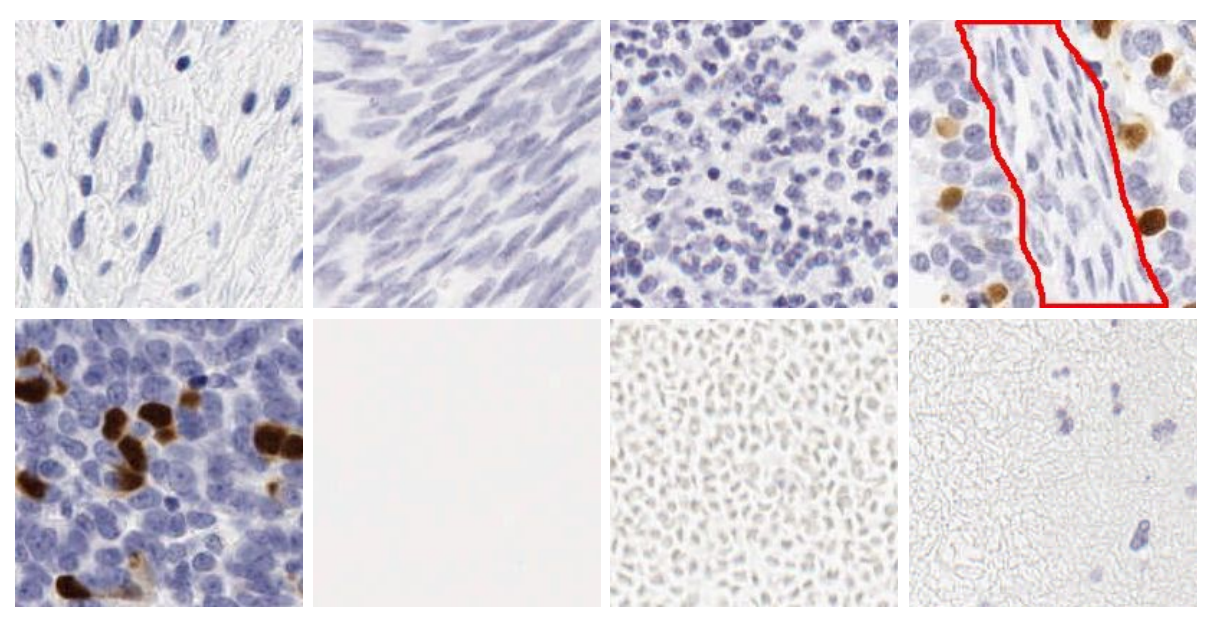

Fig. 2. First row (from the left to the right), various types of stroma: loose connective tissue, cellular stroma, inflammatory cells, and fibrous connective tissue (surrounded area). Second row: other types of compartments encountered in an image: Epithelial cancer cells, Background, Red Blood Cells, Mucus.

We propose a high resolution analysis strategy. The image's volume (3 to $10 \mathrm{~GB})$ prevents to process $V S$ at once. The tempting solution is to process tiles of a splitted image. But this implies hard constraints on elementary image edges, especially when managing large structures that can be spread over several tiles. Furthermore, large scale structure segmentation can imply local as well as global characteristics which are difficult to take into account. The proposed multiscale approach consists in selecting, at various scales, regions of interest containing objects to be further segmented. This allows the user to adjust the decomposition of the image to its contents. A large structure will be segmented at a low resolution (subsampled image size is then small enough to be held in memory, so that there will be no edge effect to manage). The result of this segmentation will serve as a mask for segmenting a smaller structure at a higher resolution. The procedure can be reiterated until localization of tiny structures inside the full resolution virtual slide.

We used wavelet transform [5] to subsample the images. A first partitioning between background and tissue can be done at a very low resolution $(32 \mu \mathrm{m})$. For this purpose, the image histogram was regarded as a mixture of three gaussian curves (one mode for background, and two closer modes for stroma and epithelium). Parameters of these three gaussian curves can be estimated by an histogram analysis. The likelihood of a pixel to belong to a class (background or tissue) can be computed and the image can then be segmented by maximum of likelihood. The resulting binary mask of tissue compartment was improved thanks to mathematical morphology operations (hole fill and geodesic opening).

The HMT model was applied to tissue compartment using marginal statistics of wavelets coefficients. 


\subsection{Hidden Markov Tree (HMT) Model}

At each scale, the value of coefficients of a wavelet transform depends on the regularity of the signal. A singularity would yield a large coefficient of wavelets that could cascade through scales, while an area with small variation values would yield a series of small coefficients. These properties of the wavelet transform mean that there is a small number of large coefficients bringing the mainpart of the energy of the signal, and many small coefficients. To approximate both the marginal and joint wavelet coefficients statistics, Crouse 4 proposed to use a model of Gaussian mixture applied to wavelet coefficients in each sub-band, and a hidden Markov tree (HMT). The HMT model associates to each wavelet coefficient a (hidden) state variable that controls whether it is Large (L) or Small (S). The joint density of each coefficient is then modeled as a gaussian mixture with a high variance and null average gaussian for the $\mathrm{L}$ state and with a low variance one for the $\mathrm{S}$ state. The model captures inter-scale dependencies between the wavelet coefficients using a Markov chain, with one dependency between the resolution levels [Fig 3 .

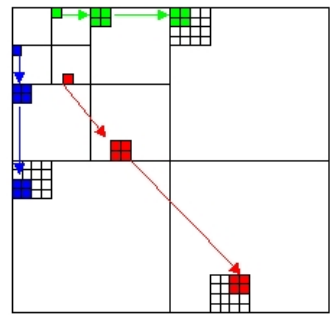

(a)

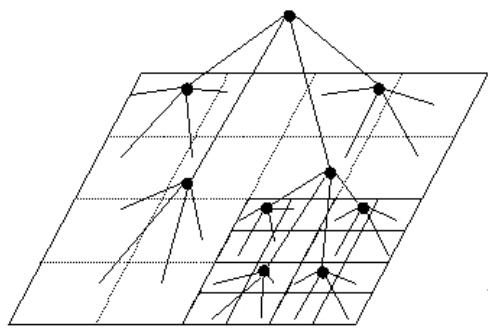

(b)

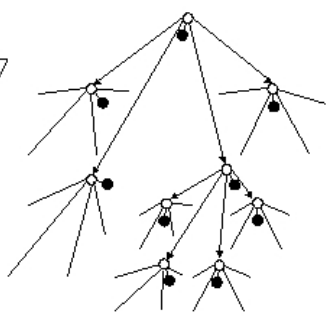

(c)

Fig. 3. Drawing from [9] a) The father-son dependencies of WT sub-bands. (b) The quad-tree structure of the model, detailed for a sub-band. (c) The HMT model, detailed for a sub-band. Each wavelet coefficient (black node) is modeled by a gaussian mixture controlled by a hidden state (white node).

This model is used by Choi 9 to achieve the segmentation of textured images. The method consists in three phases: a learning phase, a phase of segmentation at each level and a phase of inter-scales merging. During the learning phase, the model parameters $\mathcal{M}$ are learnt for each texture, through an EM (Expectation Maximization) algorithm suited to HMT [4: for each state (L or S), each level and each sub-band, the parameters of the representative Gaussian shapes (mean, variance and probability of occurrence) are learnt, as well as the parameters of transition from one state to another, between two resolutions levels:

$\left[\begin{array}{cc}\varepsilon_{s=S}^{f=S} & \varepsilon_{s=L}^{f=S} \\ \varepsilon_{s=S}^{f=L} & \varepsilon_{s=L}^{f=L}\end{array}\right]=\left[\begin{array}{cc}\varepsilon_{s=S}^{f=S} & 1-\varepsilon_{s=S}^{f=S} \\ 1-\varepsilon_{s=L}^{f=L} & \varepsilon_{s=L}^{f=L}\end{array}\right]$ where $\varepsilon_{s=b}^{f=a}$ represents the probability that the father is in state $a$ while the son is in state $b$. 
During the phase of segmentation, the likeness between the data of the wavelets transform of the observed image and the HMT model of learnt images is assessed by calculating a likelihood function :

$$
f\left(\mathcal{T}_{i} \mid \mathcal{M}\right)=\prod_{\text {orientations }} \sum_{m=S, L} f\left(\mathcal{T}_{i} \mid S_{i}=m, \mathcal{M}_{o}\right) \times p\left(S_{i}=m \mid \mathcal{M}_{o}\right)
$$

where $\mathcal{T}_{i}$ is a sub-tree of root $w_{i}$ in one of the sub-bands, $\mathcal{M}_{o}$ represents the model parameters for a particular orientation and $S_{i}$ is the hidden state of $w_{i}$. Each pixel is then affected, with the Maximum of Likelihood method, to the more similar texture. This yields to a "raw" segmentation at each level.

To improve this first segmentation, the segmentations at different resolutions are subsequently merged. This merging is achieved by maximizing the likelihood of a pixel to belong to a class, taking into account both its value in the raw segmentation and a context vector. This context vector can correspond to the value of the father and of the neighbors of the father [9], of the neighbors of the son, or a combination of several context vectors [10] [Fig 4].
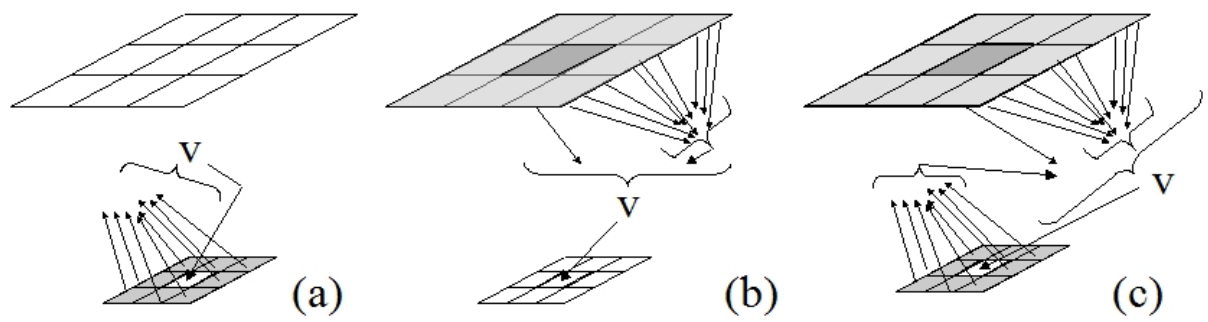

Fig. 4. Drawing from [10]. The context vectors $V$ used: a) neighbors of the son (b) neighbors of the father and the fathers value (c) neighbors of the father, sons and fathers value.

\subsection{Selection of Hyper-parameters}

The HMT parameters are calculated through a learning set of pure class images, for each combination of the method parameters. Indeed, the HMT model itself can be parameterized : one have to choose the wavelet base (and possibly its order), the color image component on which the WT is applied and the number of resolution levels on which the analysis is focused. These four hyper-parameters can greatly affect the HMT model results. Each set of hyper-parameters allows to generate a different segmentation.

Each set of hyper-parameters coresponds to a classifier, and the best of them have to be selected thanks to an evaluation of their performances. Rather than compare them for all classes together, the multiclass problem is transformed into a set of binary ones by decomposing it in one-against-one problems [11. To evaluate the classifiers, each one is trained to become a discriminative specialist in two classes (dichotomizer). For this purpose, a base containing images with 
two classes solely is used. HMT segmentation is realized by proposing exclusively these two classes as a possible result of segmentation. The classifier kept for distinguishing the two classes, is the one that maximizes the proportion of well segmented pixels on the two classes compared to the manual drawn "ground truth". The proportion of well segmented pixels, for each class, represents the confidence attached to the classifier thereafter. There is so $N=n *(n-1) / 2$ binary classifiers (where $n$ is the number of classes). Each classifier uses parameters learned by the HMT model for one set of hyper-parameters.

\subsection{Merging of Each Classifier Segmentation}

To merge the outputs of the $N$ classifiers, many combination rules can be applied to couple the estimates of each binary classifier in order to obtain class membership estimates for the multiclass problem.

The most commonly used combination rule is probably the Majority Vote 12. Class membership estimates are computed according to the formula:

$p\left(\omega=\omega_{i} \mid x\right)=\frac{1}{N} \sum_{j=0, j \neq i}^{N} I\left(\Psi_{\omega_{i}, \omega_{j}} \mid x\right) \times C f\left(\omega_{i}, \Psi_{\omega_{i}, \omega_{j}}\right)$ where $\Psi_{\omega_{i}, \omega_{j}} \mid x$ is the probability of segmentation result (class $\omega_{i}$ or $\omega_{j}$ ) with classifier $\Psi_{\omega_{i}, \omega_{j}}$ at pixel $x$. $I\left(\Psi_{\omega_{i}, \omega_{j}} \mid x\right)=1$ if $\Psi_{\omega_{i}, \omega_{j}} \mid x=\omega_{i}$ and 0 otherwise. $C f\left(\omega_{i}, \Psi_{\omega_{i}, \omega_{j}}\right)$ is the confidence in $\Psi_{\omega_{i}, \omega_{j}}$ for class $\omega_{i}$. The resulting label is computed as $\operatorname{argmax}(p(\omega \mid x))$.

One can also use combination rule based on Error Correcting Output Codes (Ecoc) [13. Each class is represented by its own output code in the output vector space. The choosen class is the one that is the closest to the $0 / 1$ prediction vector obtained from the classifiers. The Loss Based Decoding $(L B D)$ [14 is an improvement of Ecoc because it allows to use directly the outputs of the binary classifiers instead of their hard $0 / 1$ predictions.

\section{Experimentation and Results}

The tests have been conducted with several wavelets bases (Haar, Battle Lemarie, Daubechies and bi-orthogonal Splines). Two orders of wavelets (a low one and a high one) were used when possible and one to five levels of HMT decimation (resulting in four levels of segmentation) were computed. WT was computed on red, green, blue and luminance components as well as on excess red image (twice red minus green and blue), on excess green image and on excess blue image. Scheunders representation was also used. Scheunders [15] proposed a method to construct a wavelet representation combining the color plans calculating, based on the three values, a coefficient reflecting their relative importance. These parameters constitute a total of 192 different segmentations. HMT training set consists of five $512 \times 512$ images of each class. Classifiers are selected on an other training set of four 1024x1024 images per pair of class (i.e. 40 images).

The training set was improved several times, to take into account some variability within the same class, until its images are well segmented by the process.

The resulting hyper-parameters after selection are summarized in Table 1. 
Table 1. Parameters allowing good discrimination of compartments. (Epi stands for cancer epithelial cells, CS for cellular stroma, Back for background, LCT for loose connective tissue and IS for inflammatory stroma).

\begin{tabular}{|c|c|c|c|c|c|c|c|}
\hline \multicolumn{2}{|c|}{ Compartment } & Wavelets & Wavelets & Component & $\begin{array}{c}\text { Level of } \\
\text { decimation }\end{array}$ & \multicolumn{2}{c|}{$\begin{array}{c}\text { Confidence in } \\
\text { compart. 1 }\end{array}$} \\
compart. 2 \\
\hline Epi & Back & Haar & $/$ & Scheunders & 1 & 99.81 & 99.84 \\
Epi & LCT & Haar & $/$ & Scheunders & 2 & 98.91 & 93.32 \\
Epi & CS & Splines & 5 & Xred & 4 & 81.88 & 85.76 \\
Epi & IS & Battle & 1 & Xgreen & 3 & 98.25 & 92.83 \\
Back & LCT & Battle & 1 & red & 2 & 98.95 & 99.65 \\
Back & CS & Haar & $/$ & Scheunders & 1 & 99.80 & 99.57 \\
Back & IS & Haar & $/$ & blue & 2 & 99.07 & 99.53 \\
LCT & CS & Haar & $/$ & red & 2 & 94.07 & 98.13 \\
LCT & IS & Daubechies & 10 & Xgreen & 4 & 96.30 & 98.98 \\
CS & IS & Daubechies & 2 & Xgreen & 3 & 97.48 & 90.56 \\
\hline
\end{tabular}

Various combination rules were tested to merge the binary classifiers : Error Correcting Output Codes , Loss Based Decoding, but they did not provide a significant improvement over Majority Vote.

The testing set is composed of fifteen 2048x2048 images. Compared to reference manual segmentation, 60.05 to 89.67 percent of well labeled pixels are obtained together with correctly localized borders [Fig [5]. Average is $71.02 \%$. The segmentation result has been improved with further spatial regularization to ban tiny area. The main default is that in some cases, epithelium may be confused with cellular and inflammatory stroma (the closest compartments).

Tests are conducted on one processor of a $3 \mathrm{GHz}$ Xeon quadriprocessor. Processing time is from ten minutes to two hours per image. Mean time is half an hour. That is about one hundred to three hundred hours for the whole VS, depending of the amount of background.

\section{Conclusion and Prospects}

In this paper, we have investigated a strategy for segmenting various stromal compartments on ovarian carcinoma virtual slides. We proposed to use a wavelet domain hidden Markov tree model to get classifiers. Selection among these classifiers is done with a one against one approach. Classifiers outputs combination was performed thanks to a Majority Vote rule. Promising results are obtained on few selected test images and should be confirmed on a larger test set. The ratio of well classified pixels obtained must be appraised taking into account the fact that the transitions between regions of stroma are not straightforward and sometimes difficult to identify even for an expert. However, some mislabeling of the epithelium may happen, and processing time (about one hour for a 2048x2048 image) due to Expectation Maximization algorithm's convergence is prohibitive for any clinical use. 

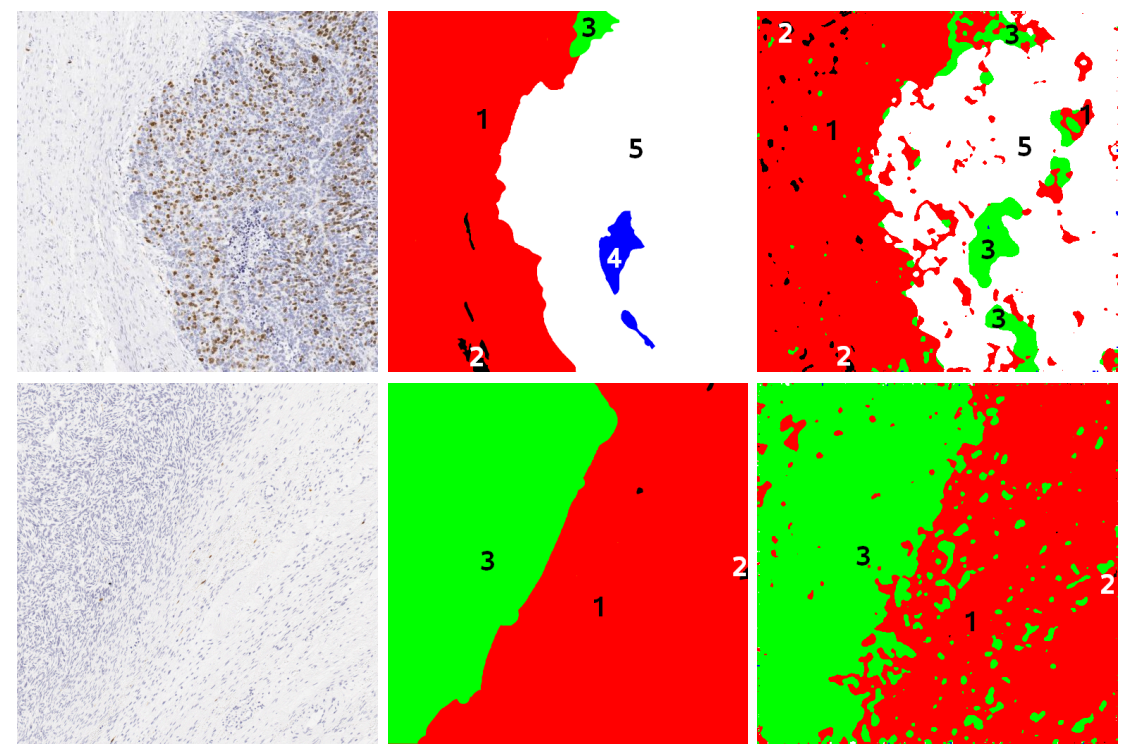

Fig. 5. Left : image to be segmented, middle : manual segmentation, right : automated segmentation. 1 is LCT, 2 is Back, 3 is CS, 4 is IS, and 5 is Epi.

To answer the problem of cancer cells foci detection, we will use previously described methods [8] to locate epithelium at low resolution and eliminate it from the higher resolution analysis.

To cope with the processing time, we have to optimize our code and consider replacing EM part with a faster converging method. We also plan to study other segmentation approaches including image patch exemplar based methods.

\section{Acknowledgments}

This work was funded by a BDI grant cofinanced by the CNRS and the Regional Council of Lower Normandy.

\section{References}

1. Wang, Y., Turner, R., Crookes, D., et al.: Investigation of methodologies for the segmentation of squamous epithelium from cervical histological virtual slides. In: IMVIP 2007, Int. Machine Vision and Image Processing Conference, pp. 83-90 (2007)

2. Pan, T.C., Mosaliganti, K., Machiraju, R., Cowden, D., Saltz, J.H.: Large scale image analysis and nuclear segmentation for digital microscopy images. In: APIII, Frontiers in Oncology and Pathology Informatics, Pittsburgh (2004)

3. Dombre, J.: Systèmes de représentation multi-échelles pour l'indexation et la restauration d'archives médiévales couleur. PhD thesis, University of Poitiers, France (2003) 
4. Crouse, M.S., Nowak, R., Baraniuk, R.: Wavelet-based statistical signal processing using hidden markov models. IEEE Trans. Signal Processing 46, 886-902 (1998)

5. Mallat, S.: A wavelet tour of signal processing. Academic Press, London (1999)

6. Elie, N., Labiche, A., Michels, J.J., Herlin, P.: Control of low resolution scanning of ovarian tumor stromal compartment. Image Analysis and Stereology 24(2), 85-93 (2005)

7. Tran, T., Elie, N., Plancoulaine, B., et al.: An original approach for quantification of blood vessels on the whole tumour section. Analytical cellular pathology 25(2), 63-75 (2003)

8. Elie, N., Plancoulaine, B., Signolle, J.P., Herlin, P.: Simple way of quantifying immunostained cell nuclei on the whole histologic section. Cytometry 56A(1), 37$45(2003)$

9. Choi, H., Baraniuk, R.: Multiscale image segmentation using wavelet-domain hidden markov models. IEEE Trans. on Image Processing 10(9), 1309-1321 (2001)

10. Fan, G., Xia, X.G.: A joint multicontext and multiscale approach to bayesian segmentation. IEEE transactions on geoscience and Remote Sensing 39(12), 2680-2688 (2001)

11. Hastie, T., Tibshirani, R.: Classification by pairwise coupling. Annals of statistics 26(2), 451-471 (1998)

12. Friedman, J.H.: Another approach to polychotomous classification. Technical report, Department of Statistics, Stanford University (1996)

13. Dietterich, T.G., Bakiri, G.: Solving multiclass learning problems via errorcorrecting output codes. Journal of Artificial Intelligence Research 2, 263-286 (1995)

14. Allwein, E.L., Schapire, R.E., Singer, Y.: Reducing multiclass to binary: A unifying approach for margin classifiers. Journal of Machine Learning Research 1, 113-141 (2000)

15. Scheunders, P.: An orthogonal wavelet representation of multivalued images. IEEE Transactions Image Processing 12(6), 718-725 (2003) 